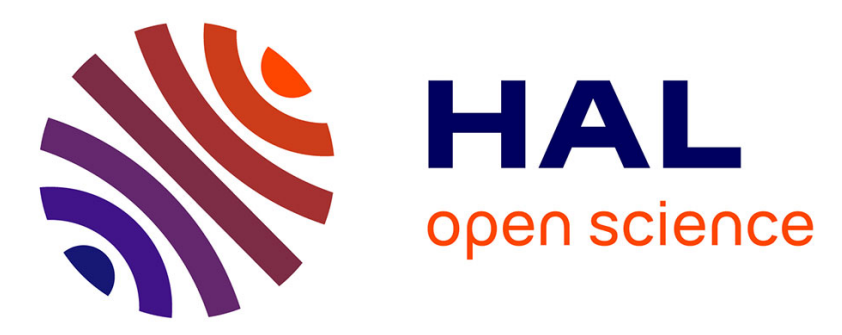

\title{
Stress state influence on nonlocal interactions in damage modelling
}

Cédric Giry, Frédéric Dufour, J. Mazars, Panagiotis Kotronis

\section{To cite this version:}

Cédric Giry, Frédéric Dufour, J. Mazars, Panagiotis Kotronis. Stress state influence on nonlocal interactions in damage modelling. EURO-C 2010, Computational Modelling of Concrete Structures, Mar 2010, Rohrmoos/Schladming, Austria. pp.145-152. hal-01008966

\section{HAL Id: hal-01008966 https://hal.science/hal-01008966}

Submitted on 5 Nov 2019

HAL is a multi-disciplinary open access archive for the deposit and dissemination of scientific research documents, whether they are published or not. The documents may come from teaching and research institutions in France or abroad, or from public or private research centers.
L'archive ouverte pluridisciplinaire HAL, est destinée au dépôt et à la diffusion de documents scientifiques de niveau recherche, publiés ou non, émanant des établissements d'enseignement et de recherche français ou étrangers, des laboratoires publics ou privés. 


\title{
Stress state influence on nonlocal interactions in damage modelling
}

\author{
Cédric Giry, Frédéric Dufour \& Jacky Mazars \\ Grenoble Institute of Technology/UJF/CNRS Lab 3S-R, BP 53, 38041 Grenoble Cedex 9, France
}

Panagiotis Kotronis

Ecole Centrale de Nantes, Institut GeM, UMR 6183 CNRS, 1 Rue de la Noe, F-44321 Nantes, France

This paper presents a modification of an integral nonlocal damage model used to describe concrete behaviour. It aims at providing a better treatment of areas close to a boundary and a fracture process zone where the interactions between points should vanish. Modifications on the original integral nonlocal model are introduced by considering the stress state of points in the weight function used to compute the nonlocal variables. Computations show that local information such as strain or damage profiles are significantly different, leading to a narrower region where damage equal to 1 in the case of the modified nonlocal model. It allows to better approach a discontinuity of the displacement field upon failure and thus, improves the estimation of the crack opening that has been developed in post-processing for this type of calculation.

\section{INTRODUCTION}

Damage models have been widely developed and used these last decades to describe the behaviour of concrete structures. Due to strain softening, these models sometimes involve media with microstructure ("local" second gradient models) (Chambon et al. 2001), (Kotronis et al. 2005) or more often nonlocality that is introduced through integral (PijaudierCabot and Bažant 1987) or gradient (Peerlings et al. 1996) regularisation techniques. Allowing retrieving the objectivity of the results, the nonlocal approach can find its origins in the micromechanics of cracks interaction (Bažant 1994). However, there are still some pending issues.

The nonlocal interactions are set constant all along calculation leading to unacceptable damage profile at the end. A damage equal to 1 corresponds actually to a crack and should be concentrated on a line, whereas a band is obtained. Moreover, in the vicinity of a boundary, the weight function is chopped off and normalised. This results to a damage field preferably developed near the boundaries. It has already been pointed out that the regularisation technique should be altered close to boundaries (Krayani et al. 2009). Besides and in a more general sense, the apparition of a crack can be seen as the creation of a new boundary and thus, the interactions between points on both sides of this crack should vanish. A crack separates two areas that can no more interact with each other.
As the nonlocality is the influence of a point on an other point, it is clear that the nonlocal model should evolve when cracks initiate. For the original nonlocal model, these two points still interact. As a consequence, a new parameter should be introduced in the modelling reflecting the state of each point regarding the nonlocality.

Several workers have proposed evolution law for the internal length, parameter governing the area of interaction, or equivalent parameter regarding the method of regularisation used.

Geers and coworkers proposed (Geers et al. 1998) a direct coupling between the material length parameter and the local strain state of the material leading to a transient behaviour of the nonlocal effect and a localisation of the strain at high state of damage.

Voyiadjis and Abu Al-Rub gave (Voyiadjis and Abu Al-Rub 2005) for gradient plasticity theory, an evolution equation for the length scale parameter depending on several parameters among which the plastic strain. This equation, based on experimental observations, leads to a decrease of the length scale parameter with the plastic strain.

Desmorat and Gatuingt proposed (Desmorat and Gatuingt 2007) also an interesting aspect to improve the original integral nonlocal model. Instead of using an internal length to treat the interaction between two points, they introduced an internal time corresponding to the time needed for a wave to propagate from point 
$\mathrm{x}$ to point s. More particularly, it allows to reduce the interactions between two points across a crack as the wave has to turn around the crack to go from one point to the other. In spite of its direct physical considerations, this approach seems to be prohibitive regarding the computational time.

In the same way, Pijaudier-Cabot and Dufour replace (Pijaudier-Cabot and Dufour 2010) the geometrical distance between two Gauss points used in the weight function by a distance that take into account the state of damage between these two points. This method, showing good results for 1D problem, can however present some difficulties in terms of computation when 2D or 3D specimen are studied. Indeed, the integration of damage along a path is not trivial to calculate.

Bažant has developed (Bažant 1994) also an evolution of the shape of the domain of interaction depending on the state of stress and the location of the two points considered. These developments, based on micromechanics of cracks interaction, give an anisotropic additional nonlocal term to the original nonlocal parameter. In this development, the stress state is introduced in the regularisation however, a nonlocal term remains during all the calculation avoiding the possibility for a point to become local when damage evolves.

For local second gradient models, the width of the localized zone can evolve freely (Kotronis et al. 2008): for a quasi-brittle (bi-linear) constitutive law the localization zone stays constant, whereas for a ductile (softening) constitutive law it decreases. A simple 1D damage model able to control a priori the evolution of the localisation zone has been proposed recently (Kotronis 2008).

In the present paper, we propose an evolution of the regularisation that yields a decrease of interaction during the nonlinear process, as proposed by the different authors quoted previously, and depending on the state of stress in an approach similar as the one developed by Krayani and coworkers (Krayani et al. 2009). First, we present the original non local damage model. Then, we introduce the modifications according to the the previous remarks. The performance of the model is finally illustrated studying the case of a bar under tension.

\section{MODELS FOR DAMAGE AND POST- PROCESSING FOR CRACK OPENING}

We recall hereafter the main equations involved in the nonlocal damage model considered in this study (Mazars 1986; Mazars and Pijaudier-Cabot 1996). Then, a modification of the weight function is proposed in order to deal with the problems mentioned previously.

\subsection{Damage model}

The scalar isotropic damage model developed by Mazars for describing the non linear behaviour of concrete under monotonic loading is used (Mazars 1986).

The general stress-strain relationship is:

$$
\sigma_{i j}=(1-D) C_{i j k l}: \varepsilon_{k l}
$$

$\sigma_{i j}$ and $\varepsilon_{k l}$ are the components of the Cauchy stress and strain tensors, respectively ( $\mathrm{i}, \mathrm{j}, \mathrm{k}, \mathrm{l} \in[1,3]$ ) and $C_{i j k l}$ are the components of the fourth-order elastic stiffness tensor. D is a damage scalar variable quantifying material degradation that grows from zero (virgin material) to one (completely degraded material).

Damage is determined as a linear combination of two damage variables (equation 2): $\mathrm{D}_{t}$ and $\mathrm{D}_{c}$ which are damage due to tension and compression respectively:

$$
D=\alpha_{t} D_{t}+\alpha_{c} D_{c}
$$

The parameters $\alpha_{t}$ and $\alpha_{c}$ depend on the stress state. The damage evolution is characterised by the following law.

$$
D_{c, t}=1-\frac{\varepsilon_{D 0}\left(1-A_{c, t}\right)}{Y}-\frac{A_{c, t}}{\exp ^{\left[B_{c, t}\left(Y-\varepsilon_{D 0}\right)\right]}}
$$

$\mathrm{A}_{t}, \mathrm{~B}_{t}, \mathrm{~A}_{c}$ and $\mathrm{B}_{c}$ are the parameters governing the shape of the evolution law. $\varepsilon_{D 0}$ is the strain threshold for the initiation of damage. Classical values can be found in (Mazars 1986).

The state variable $\mathrm{Y}$ definition is:

$$
Y=\max \left(Y, \varepsilon_{e q}\right)
$$

with $Y=\varepsilon_{D 0}$ initially, and the equivalent strain $\varepsilon_{e q}$ defined by Mazars as:

$$
\varepsilon_{e q}=\sqrt{\sum_{i=1}^{3}\left\langle\varepsilon_{i}\right\rangle_{+}^{2}}
$$

$\left\langle\varepsilon_{i}\right\rangle_{+}$denotes the positive parts of the principal strains.

This constitutive relation exhibits strain softening. As a consequence, a regularisation technique should be used in order to avoid mesh dependency and illposedness of the governing equations of equilibrium. Among the existing techniques, we can quote the integral nonlocal or gradient enhanced models (see e.g. (Bažant and Jirasek 2002)). An internal length is added with these models allowing to dissipate energy in a non zero area. The developments made in the present work deal with the integral nonlocal model. 


\subsection{Classical integral nonlocal model}

In the nonlocal damage model, the equivalent strain given by the equation 5 is replaced by an average equivalent strain $\bar{\varepsilon}_{e q}$ over a volume $\Omega$ in the equation governing the growth of damage as defined by Pijaudier-Cabot (Pijaudier-Cabot and Bažant 1987).

$$
\bar{\varepsilon}_{e q}(\boldsymbol{x})=\frac{\int_{\Omega} \phi(\boldsymbol{x}-\boldsymbol{s}) \varepsilon_{e q}(\boldsymbol{s}) d \boldsymbol{s}}{\int_{\Omega} \phi(\boldsymbol{x}-\boldsymbol{s}) d \boldsymbol{s}}
$$

$\phi(x-s)$ is the weight function defining the interaction between the point located at $\boldsymbol{x}$ considered and the neighbouring points located at $s$ inside the volume of the structure $\Omega$. This formulation fulfils the requirement of non alteration of an uniform field. The basic nonlocal weight function is often taken as the polynomial bell-shaped function or here as the Gauss distribution function:

$$
\phi(x-s)=\exp \left(-\left(\frac{2\|x-s\|}{l_{c}}\right)^{2}\right)
$$

where $l_{c}$ is the internal length of the model and $\|x-s\|$ the distance between points located at $x$ and $s$.

The point located at $s$ (distributing point) influences its neighbourhood equivalently in all directions and during all the calculation (figure 1 and 2,b)) except close to boundaries. The shape of the Gaussian function depends only on the initial internal length. As a consequence, the area close to boundaries are not correctly taken into account. It can be observed an attraction of the damage by the boundaries due to the truncation of the volume of interaction (Krayani et al. 2009). Considering a point at the level of the boundary, the number of distributing points with an influence non negligible on it is divided by two. As a consequence, the term at numerator in equation 7 is also divided by two, leading thus to a double amplitude of the interactions close to the boundary. A first proposal was made by the authors to avoid this phenomenon by considering an evolution of the domain of regularisation in the vicinity of free boundaries.

Another problem that has been pointed out, is the treatment of the fracture process zone at high damage level. On figure 2b), quarter of a plate with a central crack is represented. It is submitted to tension of equal magnitude in both directions. The circles given on the figure represent isovalues of the Gaussian function, corresponding to the influence of the point located at the center of these circles. In the classical nonlocal model, this point can influence a point on the other side of the crack as only the distance between them is considered in the model.

However, the interaction should vanish as soon as the fracture process zone passes between the two points considered. A solution to this problem can be

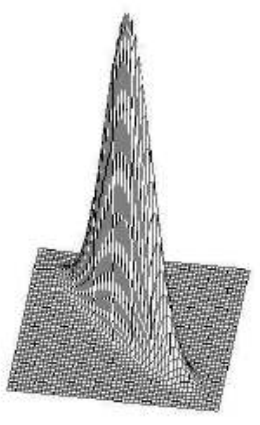

a)

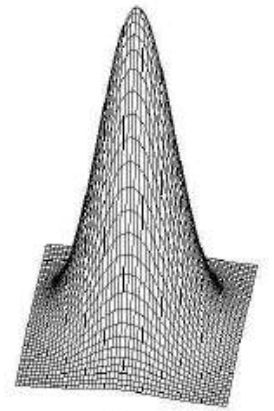

b)
Figure 1: Influence of a distributing point. Gaussian function. (a) modified nonlocal model, b) original nonlocal model)

to define an evolution of the interaction that should decrease in the vicinity of a cracked area.

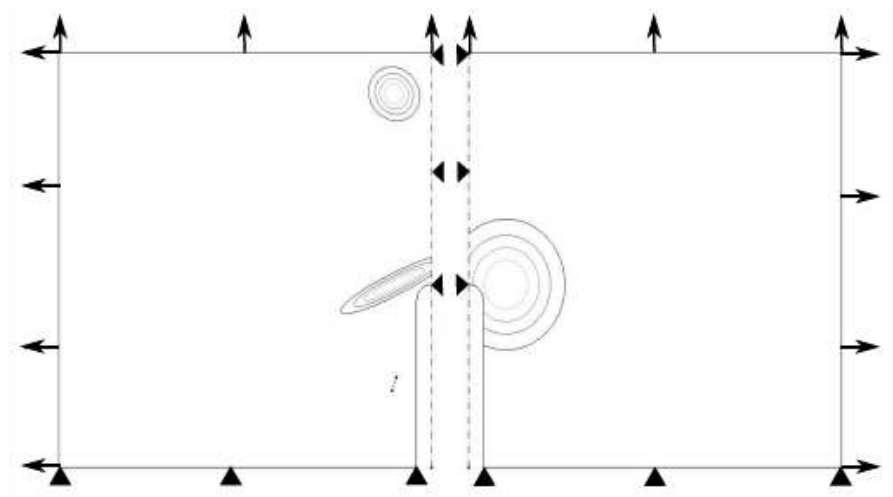

a)

b)

Figure 2: Isovalues of the influence of different points in the specimen (a) modified nonlocal model, b) original nonlocal model)

\subsection{Modified integral nonlocal model}

In this paper, we propose to modify the integral nonlocal regularisation in order to deal with the problems quoted previously. We restrict the domain of application of our modification for problems with mode I crack opening under tensile stress. During the calculation, an evolution of the interaction between points is considered through a coefficient. This coefficient depends on the stress state of the distributed points and it is introduced in the Gaussian function.

At each step, the stress state of the point located at $s$, expressed in its principal space, is known.It is denoted hereafter $\sigma_{I}(s), \sigma_{I I}(s)$ and $\sigma_{I I I}(s)$. We define an ellipsoid of center point the point located at $s$, corresponding to an homothety of the original Gaussian function with the following ratio along principal stress directions: 
$1^{s t}$ axis : $\left|\frac{\sigma_{I}(s)}{f_{t}}\right|$

$2^{\text {nd }}$ axis : $\left|\frac{\sigma_{I I}(\boldsymbol{s})}{f_{t}}\right|$

$3^{r d}$ axis $:\left|\frac{\sigma_{I I I}(s)}{f_{t}}\right|$

with $f_{t}$ the tensile strength of concrete.

The choice of $f_{t}$ leads to no modification at peak. Furthermore, the length of the axes can not overcome 1 in order to prevent high values for principal stress in compression.

In the original integral nonlocal model, the influence of point located at $s$ is isotropic. We define now an anisotropic influence of point located at $s$ depending on its stress state and the position of the point located at $\boldsymbol{x}$. The weight function now reads:

$$
\phi(\boldsymbol{x}-\boldsymbol{s})=\exp \left(-\left(\frac{2\|\boldsymbol{x}-\boldsymbol{s}\|}{l_{c} \beta\left(\boldsymbol{x}, \boldsymbol{\sigma}_{\text {prin }}(\boldsymbol{s})\right)}\right)^{2}\right)
$$

with $\beta\left(\boldsymbol{x}, \boldsymbol{\sigma}_{\text {prin }}(\boldsymbol{s})\right)$ equal to the ratio of the homothety defined previously in the direction $(\boldsymbol{s}-\boldsymbol{x})$. In 3D, we obtain for it:

$$
\beta\left(\boldsymbol{x}, \boldsymbol{\sigma}_{\text {prin }}(\boldsymbol{s})\right)^{2}=\frac{1}{\frac{\sin ^{2} \varphi \cos ^{2} \theta}{a^{2}}+\frac{\sin ^{2} \varphi \sin ^{2} \theta}{b^{2}}+\frac{\cos ^{2} \varphi}{c^{2}}}
$$

By noting $\boldsymbol{u}$ and $\boldsymbol{v}$, the vectors associated respectively to the highest and the lowest pincipal eigenvalues of the Cauchy stress tensor at the point located at $s$, we have $\theta=(\boldsymbol{u}, \boldsymbol{s}-\boldsymbol{x})$ and $\varphi=(\boldsymbol{v}, \boldsymbol{s}-\boldsymbol{x})$.

A point under high stress influences widely its neighbourhood whereas a point that encounters very low stress does not affect the surrounding points. Figure 1 (a)) gives an example of the modified Gaussian function. The coefficient $\beta$ is also calculated for point in elastic domain in order to avoid the interaction of two non damage points across a crack that encounter unloading due to the creation of the crack.

In order to avoid mathematical problems with zero stress for some principal directions and thus a zero volum ellipsoid, a minimal value for $\beta$ is fixed. Furthermore, the stress state used numerically to modify the nonlocal regularisation is the one of the last converged step thus, the model remains explicit. The general algorithm of the original nonlocal is unchanged, only the calculation of the weight function is found affected.

When we considered a point close to a free boundary, we have the following condition for its stress state: $\boldsymbol{\sigma}_{\text {prin }}(\boldsymbol{s}) \cdot \vec{n}=\overrightarrow{0}$ with $\vec{n}$ normal vector to the boundary. As a consequence, for the modified nonlocal model, this point interacts only with other points in the direction parallel to the boundary. We retrieve here the result developed in (Krayani et al. 2009) with a vanishing length of the diameter of the ellipsoid perpendicular to the boundary. The advantage of this method compared with the modification of the domain of interaction as proposed in (Krayani et al. 2009), is its adaptability to various structures. Indeed, there is no need to give the area for which the connectivity needs to be modified as it is directly taken into account through the stress state; the modification is based on mechanics rather than on geometry.

For the case of study on figure 2 (a)), we observe an evolution of the isovalues of the Gaussian function corresponding to the influence of different points in the specimen. The upper point is in a non disturbed area, as a consequence, with the prescribed loading, the isovalues are close to circles. For the middle point, in the vicinity of the crack tip, its stress state is highly disturbed and oriented, leading to ellipses for the isovalues. The lower point is shielded by the crack, as a consequence, it encounters very low stress state. In other words, it has a local behaviour and no influence on the surrounding points.

\subsection{Crack opening estimation}

A post-processing method has been developed to estimate crack opening in mode I from a continuous modelling calculation.

First, the finite element calculation is performed. Then, the location of the crack is determined by using the maximum state variable. The profile of the crack obtained is discretized in several segments. For each one, a perpendicular profile segment is defined in order to calculate the crack opening (Bottoni and Dufour 2010).

For each profile, projection of the equivalent strain by interpolation is made. Then, the method developed by (Dufour et al. 2008) to estimate the crack opening is used. Analytically, the crack is considered as a displacement jump [u] on a $1 \mathrm{D}$ profile at the location of the crack (figure 3 ).

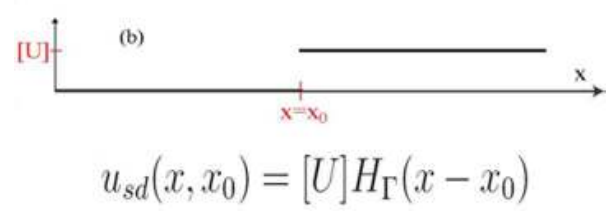

Figure 3: Displacement profile of the strong discontinuity

It corresponds to a Dirac when we consider the strain profile (figure 4).

The concept of the method is to compare the strain profile obtained numerically with the one obtained analytically by considering a strong discontinuous displacement. However, the strain profile obtained analytically corresponds to a Dirac which can be hard to use directly for comparison. As a consequence, this 


$$
\underset{\varepsilon_{s d}\left(x, x_{0}\right)=\nabla^{s} u_{s d}=[U] \delta\left(x-x_{0}\right)}{\stackrel{\text { [U] }}{\longrightarrow}}
$$

Figure 4: Strain profile of the strong discontinuity

profile as well as the one obtained numerically are regularised by means of a convolution product (figure 5).

Finally, hypothesis of equal magnitude of the strain

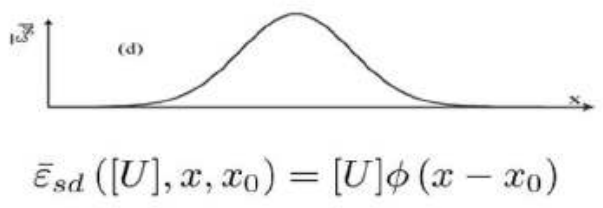

Figure 5: nonlocal strain profile of the strong discontinuity

at the location of the crack leads to an estimation of the crack opening (equation 10):

$$
[U]=\frac{\bar{\varepsilon}_{e q}\left(x_{0}\right)}{\phi(0)}
$$

An advantage of this method is to give an estimation of the error made between the analytical profile and the numerical one.

An aim of the modification made on the nonlocal regularisation is to better treat the post-peak behaviour of concrete structure. By reducing the highly damage area, we get closer to a strong discontinuity and a Dirac for the strain profile. We can expect to reduce the error as defined previously.

\section{EXAMPLE}

The test used here to illustrate the functioning of the modified nonlocal formulation is a bar under tension with a 2D geometry and a 1D loading (no Poisson's effect). In spite of its simplicity this test allows to show the change in the behaviour obtained with this improvement more particularly when dealing with high damaged state.

All the calculations presented in this paper are made with the finite element code Cast $3 \mathrm{M}$ developed by the C.E.A. (French Nuclear Agency)(wwwcast $3 \mathrm{~m}$.cea.fr/).

The bar is $1 \mathrm{~m}$ long and $0.01 \mathrm{~m}$ high (figure 6). Only one element is put on the height. We have used square elements with linear interpolation. The following parameters have been taken for the damage model describing concrete. The central element is weakened with a lower Young's modulus in order to initiate the damage in the bar.
$\mathrm{Eb}=33.7 \mathrm{GPa}$
$\mathrm{At}=1$
$v=0$.
Ebendo $=31 \mathrm{GPa}$
$B_{t}=20000$

Figure 6: Mesh of the bar

An arc-length technique is used to follow such a global response which exhibits snap-back.

\subsection{Global behaviour}

The objectivity of the results is studied. Two sizes of mesh are used (31 and 61 elements in the length of the bar). Figure 7 gives the evolution of the force versus displacement for both mesh size and nonlocal versions. We can observe the objectivity of the results for both. The small difference at the end for the modified nonlocal model is due to the fact that the smaller size mesh can more localised the damage at the end than the coarse one. This tendency is also observed in the case of gradient-enhanced damage model (Simone 2007). We observe also that the modified nonlocal approach dissipate less energy than the original one for the same initial internal length since the area of interaction (and in some way of dissipation of the energy) reduces with the decreasing stress state. The peak force is identical as we recover the value of the internal length $l_{c}$ for the modified nonlocal model.

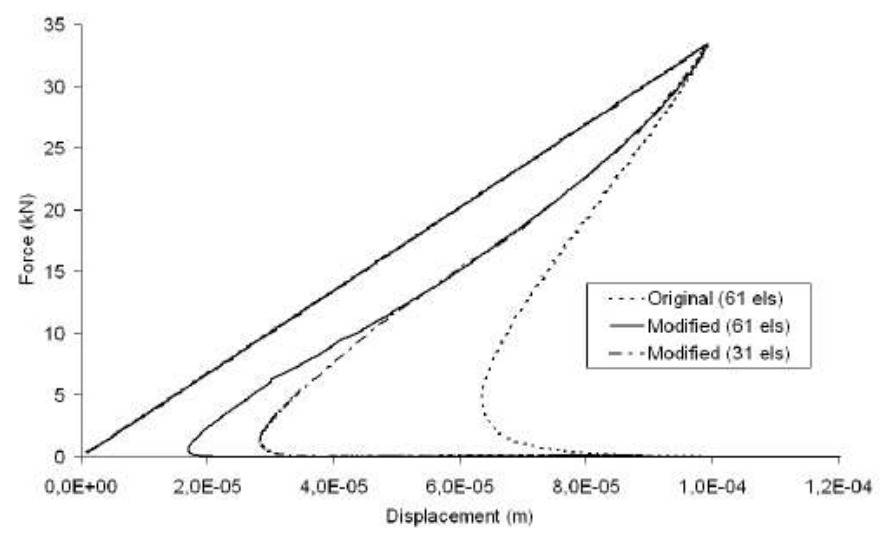

Figure 7: Evolution of the force versus displacement for the modified (31 elements: dot-and-dash line) (61 elements: continuous line) and original nonlocal models (61 elements: dash line)

\subsection{Local response}

Locally, the figures $8,9,10$ and 11 give the evolution of the strain and damage profiles along the bar for both nonlocal approaches and with 61 element in the mesh. The chosen state for the curves are from $F_{\max }$ up to $0.01 F_{\max }$ with a step of $\frac{F_{\max }}{10}$.

The classical integral nonlocal method leads to a completely damage area spread over several elements when the force vanishes. At each step, the increase of damage is observed over a wider surface. This is due 


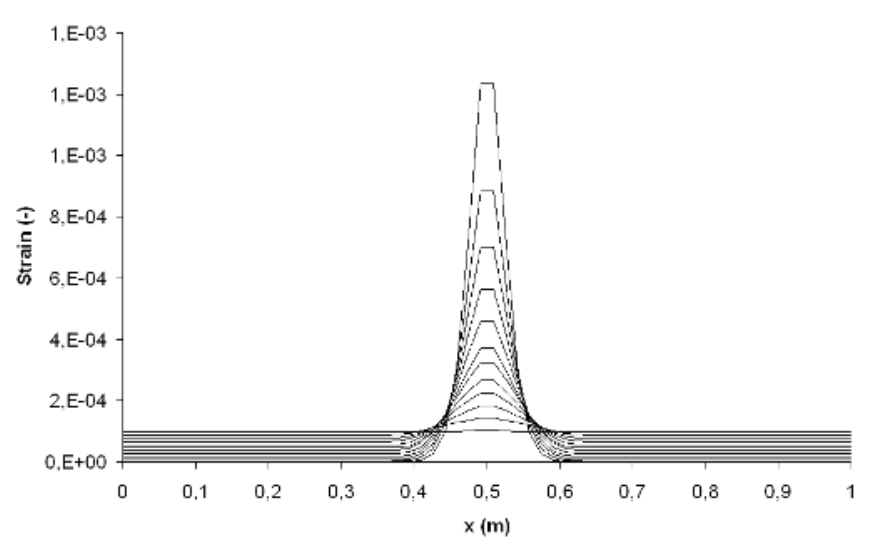

Figure 8: Evolution of the strain for the original nonlocal model

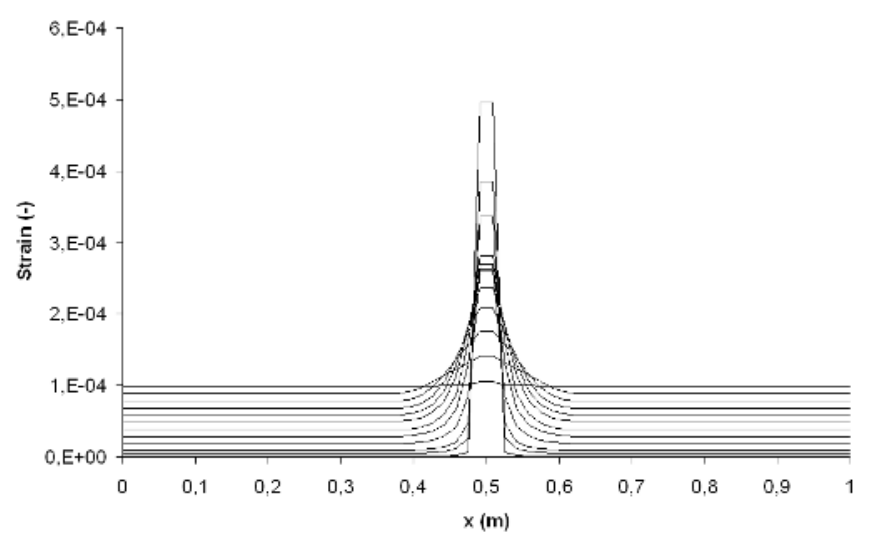

Figure 9: Evolution of the strain for the modified nonlocal model

to the fact that the interactions between Gauss points for the original nonlocal model do not evolve during the test. In reality, we can suppose only one element should encountered a damage equal to 1 whereas the surrounding elements should be partially damaged. The intensity of damage should decrease as we go further from the weakened points. Experimentally, it could be seen by measuring the evolution of density of microcracks from the main crack up to the virgin area.

The modified nonlocal model leads to more localised damage and strain profiles. The values given are projected on the nodes of the mesh and due to the linear interpolation in the elements, maximum strain and damage are reached for two nodes. Points with damage equal to one are concentrated in one element. As the Gauss points along the bar have encountered their peak stress, we observe a decrease of the interactions. Each point tends to be more local. As a consequence, the area with highest damage decreases at each step to finally be reduced to the weakened element introduce at the beginning of the calculation. For the strain field along the bar, we observe that with the modified nonlocal model, it approaches a Dirac better than with original damage model.

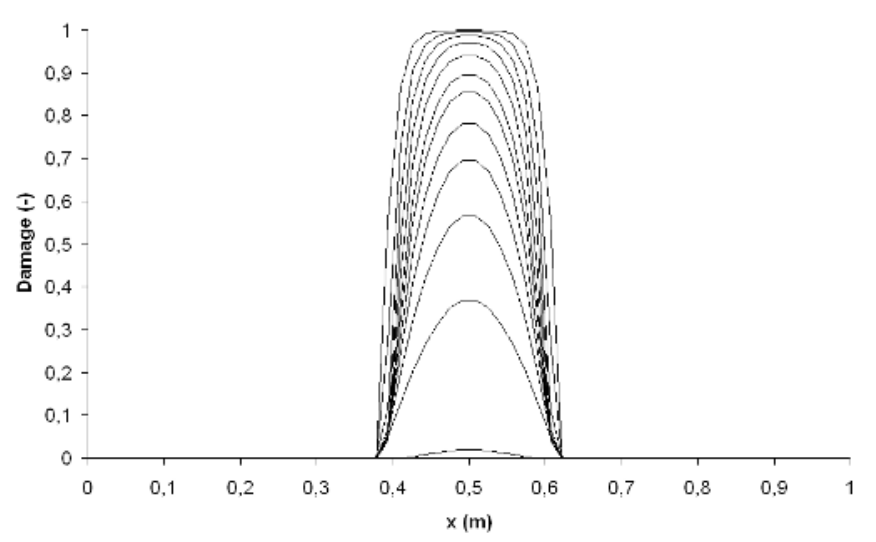

Figure 10: Evolution of the damage for the original nonlocal model

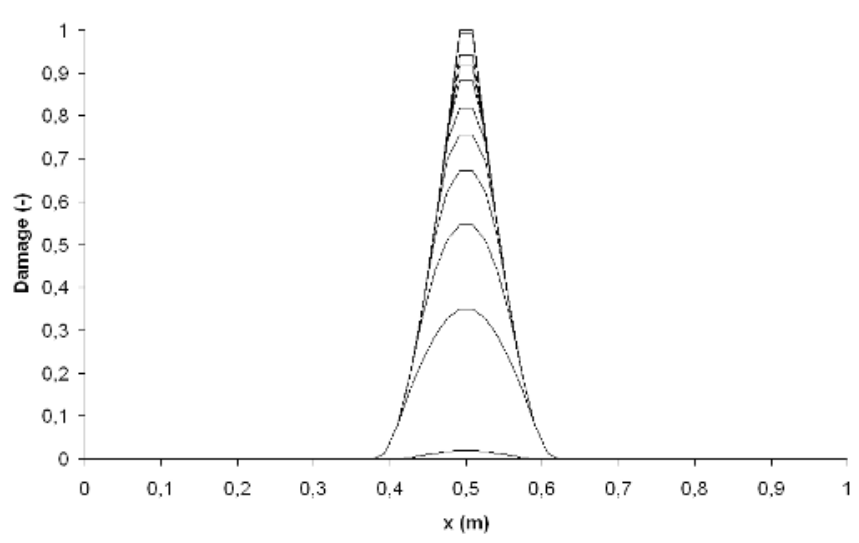

Figure 11: Evolution of damage for the modified nonlocal model

Regarding crack opening the figures 12 and 13 compare the regularised strain profile obtained from $\mathrm{FE}$ calculation and from strong discontinuity at $F=$ $0.01 F_{\text {max }}$. One can see that at this state, the modified nonlocal model gives a strain profile closer to a strong discontinuity than the original one.

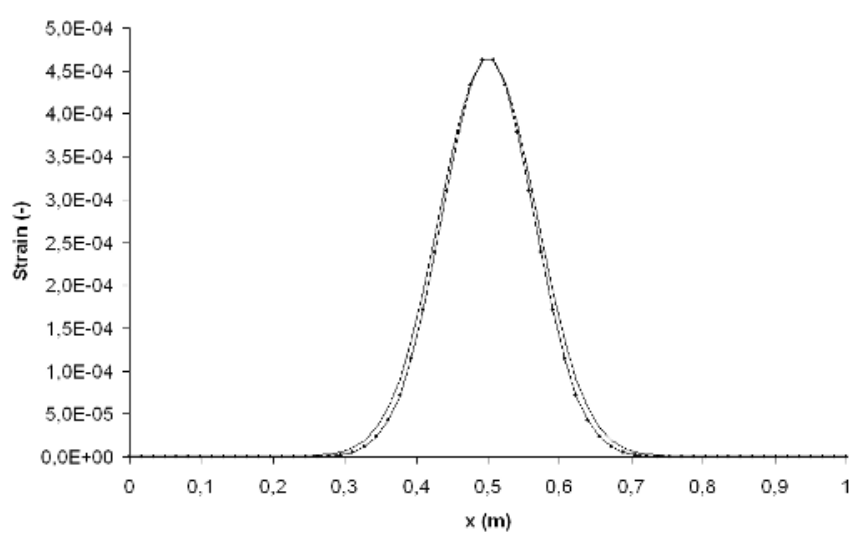

Figure 12: Regularised effective strain profiles from the strong discontinuity approach (continuous line) and from FE computations using the original nonlocal model (continuous line with points)

The figures 14 and 15 give the evolution of the 


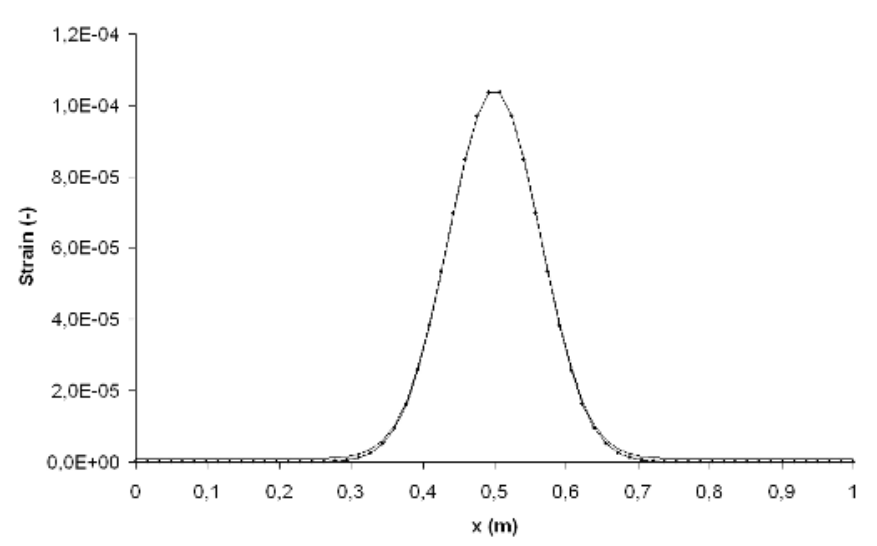

Figure 13: Regularised effective strain profiles from the strong discontinuity approach (continuous line) and from FE computations using the modified nonlocal model (continuous line with points)

crack opening in function of the displacement and the error between the regularised FE strain profile and the regularised strong discontinuity strain profile in function of crack opening.

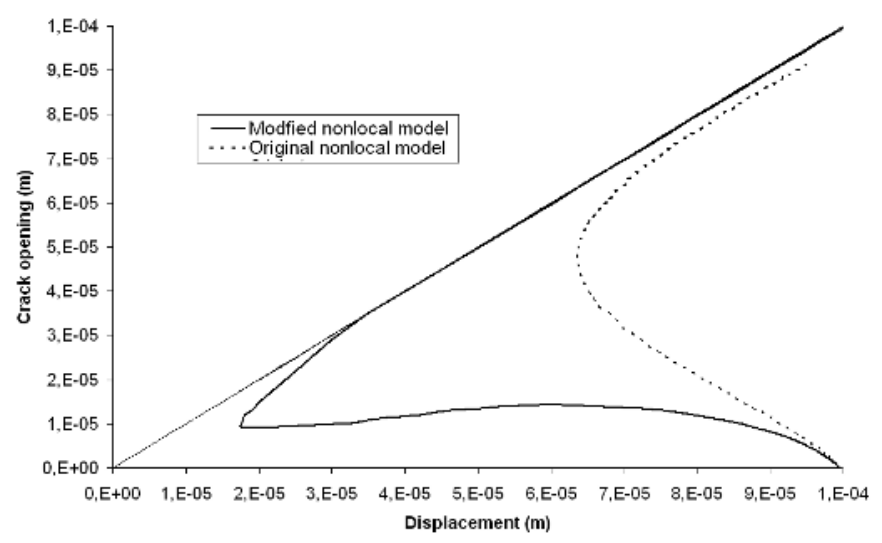

Figure 14: Crack opening as the function of the displacement (modified non local: continuous line) (original non local:dash line)

The first part of the curves correspond to the error just after the peak. In both cases, the strain field is far from a Dirac as a consequence, a relatively high error is observed. However, when the crack initiates, the error with the strong discontinuity decreases faster in the case of the modified nonlocal model validating the observations we could have made previously on the strain and damage profiles. It confirms the capability of the modified nonlocal model to approach the discontinuity without loosing the advantages of the original nonlocal model.

\section{CONCLUSIONS}

We have proposed in this paper a modification of integral nonlocal model in order to adapt the regularisation close to boundary and fracture process zone in the case of mode I failure. The stress state of each

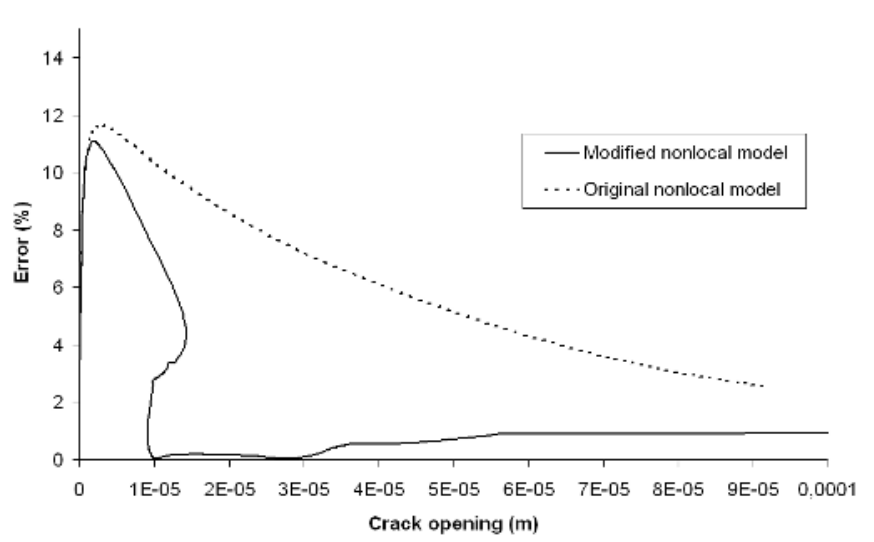

Figure 15: Relative error as the function of the crack opening (modified non local: continuous line) (original non local:dash line)

point is used during the calculation in order to create an evolution of the interaction between points. Each point interacts with its neighbourhood in function of the intensity of its principal stress values. This modification has been presented in $3 \mathrm{D}$ case. It has allowed to retrieve results given in a previous article by the $2^{\text {nd }}$ author (Krayani et al. 2009).

The modification has been illustrated through the example of a bar of concrete under tension. The objectivity of results is conserved and our proposal allows to get closer to a strong discontinuity at high damage state as the results on crack opening shows.

This last result is an important point as we can see nowadays that the information on crack opening is a hot topic. It has been shown that continuous modelling can provide this kind of information. By improving the FE calculation and more particularly by taking into account the effect of a damage zone on the neighbourhood in the calculation, we although improve the results we can provide for the crack opening.

\section{ACKNOWLEDGEMENTS}

Financial support from the Agence Nationale de la Recherche through the sustainable cities programme (contract VD08_323065)is gratefully acknowledged.

\section{REFERENCES}

Bažant, Z. P. (1994). Nonlocal damage theory based on micromechanics of crack interactions. Journal of Engineering Mechanics 120, 593617.

Bažant, Z. P. and M. Jirasek (2002). Nonlocal integral formulations for plasticity and damage: Survey of progress. Journal of Engineering Mechanics 128, 1119-1149.

Bottoni, M. and F. Dufour (2010). Topological search of the crack path from a damage-type mechanical computation. In EURO-C 2010 
Computational Modelling of Concrete Structures.

Chambon, R., D. Caillerie, and T. Matsushima (2001). Plastic continuum with microstructure, local second gradient theories for geomaterials, localization studies. International Journal of Solids and Structures (38), 8503-8527.

Desmorat, R. and F. Gatuingt (2007). Introduction of an internal time in nonlocal integral theories. Internal report LMT-Cachan, number 268, year 2007, ENS Cachan/CNRS/Université Paris 6/PRES Universud Paris.

Dufour, F., G. Pijaudier-Cabot, M. Choinska, and A. Huerta (2008). Extraction of a crack opening from a continuous approach using regularized damage models. Computers and Concrete 5(4), 375-388.

Geers, M., R. de Borst, W. Brekelmans, and R. Peerlings (1998). Strain-based transientgradient damage model for failure analyses. Computer Methods in Applied Mechanics and Engineering 160, 133-153.

Kotronis, P. (2008). Stratégies de Modélisation de Structures en Béton Soumises à des Chargements Sévères. Habilitation à Diriger des Recherches, Université Joseph Fourier, http://tel.archives-ouvertes.fr/tel-00350461/fr/.

Kotronis, P., S. Al Holo, P. Bésuelle, and R. Chambon (2008). Shear softening and localization: Modelling the evolution of the width of the shear zone. Acta Geotechnica 3(2), 85-97.

Kotronis, P., R. Chambon, J. Mazars, and F. Collin (2005). Local second gradient models and damage mechanics: Application to concrete, cd paper 5712. In ICF (Ed.), $11^{\text {th }}$ International Conference on Fracture, 20-25 March.

Krayani, A., G. Pijaudier-Cabot, and F. Dufour (2009). Boundary effect on weight function in non-local damage model. Engineering Fracture Mechanics 76, 2217-2231.

Mazars, J. (1986). A description of micro- and macroscale damage of concrete structures. Engineering Fracture Mechanics 25(5-6), 729 737.

Mazars, J. and G. Pijaudier-Cabot (1996). From damage to fracture mechanics and conversely: a combined approach. International Journal of Solids and Structures 33, 3327-3342.

Peerlings, R. H. J., R. de Borst, W. A. M. Brekelmans, and J. H. P. de Vree (1996). Gradient enhanced damage for quasi-brittle materials. International Journal for Numerical Methods in Engineering 39, 937-953.
Pijaudier-Cabot, G. and Z. Bažant (1987). Nonlocal damage theory. Journal of Engineering Mechanics 113, 1512-1533.

Pijaudier-Cabot, G. and F. Dufour (2010). Nonlocal damage model: boundary and evolving boundary effects. European Journal of Environmental and Civil Engineering (Submitted).

Simone, A. (2007). Explicit and implicit gradientenhanced damage models. European Journal of Environmental and Civil Engineering 11/78, pp.1023-1044.

Voyiadjis, G. and R. Abu Al-Rub (2005). Gradient plasticity theory with a variable length scale parameter. International Journal of Solids and Structures 42(14), 3998-4029. 